\title{
Can Persona Facilitate Ideation? A Comparative Study on Effects of Personas in Brainstorming
}

\author{
Xiantao Chen ${ }^{1}$, Ying $\mathrm{Liu}^{2}$, Ning Liu ${ }^{3}$, and Xiaojie Wang ${ }^{4}$ \\ ${ }^{1}$ Beijing University of Posts and Telecommunications, Nokia Research Center Beijing, \\ 100176 Beijing, China \\ ext-xiantao.chen@nokia.com \\ ${ }^{2}$ Nokia Research Center Beijing, 100176 Beijing, China \\ ying.y.liu@nokia.com \\ ${ }^{3}$ School of Psychology, Shandong Normal University, 250014 Jinan, China \\ sdliuning@gmail.com \\ ${ }^{4}$ Beijing University of Posts and Telecommunications, 100876 Beijing, China \\ xjwang@bupt.edu.cn
}

\begin{abstract}
Personas are results from user studies and viewed as a design and a communication tool in user-centered design processes. There were many studies addressing how to create good personas but what types of personas and how personas could help in ideation processes were less discussed in past works. In this paper, we conducted a comparative study to explore effects of personas on the ideation process and idea qualities in a brainstorming setting. The results indicated that personas could enhance the ideation process and design deliverables on two aspects: personas could help both individual designer and a group of designers focus on the target user group during the ideation process; and the delivered ideas or concepts were viewed more relevant to the user groups and were more comprehensive.
\end{abstract}

Keywords: Comparative study, Persona, Ideation, Brainstorming.

\section{Introduction}

Persona is a method introduced by Cooper to describe user profiles [1]. A well-cited definition of it is that personas are fictional archetypical characters that represent distinct groupings of behavior, goals and motivations identified in a study [2]. There were two different applications of personas in existing design practices: as a communication tool or a design tool [3]. However, little research has been done to prove the benefits of persona as a design tool.

In this research we aimed to investigate whether persona can facilitate ideation as a design tool in the early stage of a user-centered design (UCD) process. We first built three personas for middle school students in top cities of China by following Nielsen's ten steps to personas [4]. The user data that our personas were built on were from the Youth Study project. The purpose of the project was to understand their lifestyle and usage of information and communication technologies (ICTs) of urban youth in China. Based on the three personas, we conducted an exploration on effects of persona in the early concept design phases in the UCD process. Design students 
were invited to a brainstorming session and they were either presented with personas or not. We observed and coded the design process and idea qualities from the brainstorming sessions. Our results found that proper personas can help designers to deliver ideas that are more appropriate for the target user group. The study and results are presented in the rest of the paper.

\section{A Comparative Experimental Study}

Fourteen participants took part in the brainstorming session and they were divided to two groups with respectively seven people. All of them were undergraduates in industrial design and information design in a top university. For Group A, we present both three personas and raw materials including user study transcriptions and dialogues during their brainstorming session and for Group B, only raw materials were presented. The assigned topic was "Mobile solutions for senior middle school students" for both groups. And two relevant tasks were assigned at the same time. In the first task, participants were given an hour for reviewing the user study materials and generate ideas individually. In the second task, participants were asked to join a cooperative ideation session for one hour. Two final concepts needed to be sketched and delivered by each group.

Shah [5] proposed two approaches for evaluating the effectiveness of ideation: process-based measures that measure the process of ideation, and outcome-based measures relating to the results. During the experiment, all ideas created in both design tasks were recorded and analyzed. Based on the idea evaluation framework introduced by Barki [6], ten design experts were invited to evaluate ideas based on an 11-point Likert scale on a few aspects: novelty, uniqueness, perfectness, value, relevance with user needs, suitability for the users and an overall score. Since the ideas produced in the first task were quick ones with little elaborated descriptions, only novelty, uniqueness, relevance with the user group and suitability for the users of them were evaluated. About the process of brainstorming sessions, observations was conducted to track when the first idea was generated and the usage of materials.

\section{Results}

\subsection{Ideation Process}

We analyzed our observation notes in both groups. From the results, we found that the group A with personas spent less time in producing the first idea than the group B without personas. All participants in Group A generated their first ideas in the first half hour, however only two participants in Group B generated their first ideas. About the usage of materials, all participants in Group A shifted the usage of personas and raw materials to generate ideas. They thought that both of them were important to inspire novel ideas. Typical usages of personas included that they were used to get overview to target users, inspire novel ideas from the features and refine final concepts. Participants in Group A reported that details and distinct features in personas were important to inspire ideas. In Group B, several participants complained that twelve pages' raw materials were too long to read. Some of them gave up reviewing raw materials and generated ideas form their own experiences. 
Table 1. The t-test of idea quality in first task between Group A and Group B

\begin{tabular}{|c|c|c|c|c|c|}
\hline Category & & Group A & Group B & $t$ & $p$ \\
\hline \multirow{2}{*}{ Is it novel } & $M$ & 6.09 & 5.57 & \multirow{2}{*}{1.50} & \multirow{2}{*}{0.159} \\
\hline & $S D$ & 0.79 & 0.46 & & \\
\hline \multirow{2}{*}{ Is it unique } & $M$ & 5.97 & 5.47 & \multirow{2}{*}{1.49} & \multirow{2}{*}{0.162} \\
\hline & $S D$ & 0.74 & 0.48 & & \\
\hline \multirow{2}{*}{ Is it relevant with user need } & $M$ & 7.13 & 6.10 & \multirow{2}{*}{3.49} & \multirow{2}{*}{$0.004 *$} \\
\hline & $S D$ & 0.54 & 0.57 & & \\
\hline \multirow{2}{*}{ Is it suitable for user } & $M$ & 6.74 & 5.81 & \multirow{2}{*}{3.89} & \multirow{2}{*}{$0.002 *$} \\
\hline & $S D$ & 0.37 & 0.51 & & \\
\hline \multirow{2}{*}{ Overall score } & $M$ & 6.41 & 5.75 & \multirow{2}{*}{2.24} & \multirow{2}{*}{$0.045 *$} \\
\hline & $S D$ & 0.64 & 0.42 & & \\
\hline
\end{tabular}

\subsection{Idea Quality}

In the first task, participants in Group A generated an average of 6.29 new ideas per person and an average of 8.00 new ideas in Group B per person. Although Group A produced less ideas than Group B, there were significant difference on quality of ideas between the two groups according to the expert evaluation results. The t-test results indicated that idea quality of Group A is better than that of Group B on a few characteristics: relevance with user $(t(12)=3.49, p<0.005)$, suitability for user $(t(12)$ $=3.89, p<0.005)$ and overall scores $(t(12)=2.24, p<0.05)$ (see Table 1$)$. The significant differences revealed that participants in Group B inclined to produce ideas that were not suitable for the design topic. No significant difference was found on the qualities of "novelty" $(t(12)=1.50, \mathrm{~ns})$ and "uniqueness" for ideas from both groups $(t(12)=1.49, \mathrm{~ns})$. The results from the first design task showed that engaging personas in individual ideation could help participants focus on the assigned topic.

Table 2. The t-test of idea quality in second task between Group A and Group B

\begin{tabular}{llllll}
\hline Category & & Group A & Group B & $t$ & $p$ \\
\hline \multirow{2}{*}{ Is it novel } & $M$ & 6.67 & 5.94 & \multirow{2}{*}{1.92} & 0.073 \\
& $S D$ & 0.71 & 0.88 & & \\
Is it unique & $M$ & 6.39 & 5.67 & 1.62 & 0.126 \\
& $S D$ & 0.96 & 0.94 & & \\
Is it perfect & $M$ & 7.17 & 5.33 & 3.67 & $0.002^{*}$ \\
& $S D$ & 0.71 & 1.32 & & \\
Is it valuable & $M$ & 6.67 & 5.94 & 2.14 & $0.048^{*}$ \\
\multirow{4}{*}{ Is it relevant with user need } & $S D$ & 0.66 & 0.77 & & \\
\multirow{5}{*}{ Is it suitable for user } & $M$ & 7.00 & 6.06 & 2.20 & $0.042^{*}$ \\
& $M$ & 0.79 & 1.01 & & \\
Overall score & $M$ & 6.67 & 5.83 & 2.24 & $0.040^{*}$ \\
& $S D$ & 0.71 & 0.87 & & \\
& $M$ & 6.94 & 5.53 & 3.84 & $0.001^{*}$ \\
\hline
\end{tabular}


In the second design task, the two final concepts contributed in each group were evaluated. The quality of the concepts from Group A was ranked higher than that from Group B: significant difference were found on perfectiveness $(t(12)=3.67, p<$ $0.005)$, value $(t(12)=2.14, p<0.05)$, relevance with users $(t(12)=2.20, p<0.05)$, suitability for users $(t(12)=2.24, p<0.05)$ and the overall scores $(t(12)=3.84, p<$ 0.005 ) (see Table 2). The results showed that personas could facilitate the group ideation session and enable designers create complete concepts.

\section{Conclusion and Further Work}

In this research, we explored the usage of personas in early ideation processes. A comparative study proved that personas enhanced the effectiveness of brainstorming on two aspects: it helped designers to stay on the assigned design topic during the individual brainstorming session and it also helped a group of designers to agree on the topic for the group concept and delivered comparatively complete concept proposals. Moreover, personas could also potentially improve idea qualities especially on measures to indicate relevance to target users.

Although benefits of personas in brainstorming sessions were discovered, limitations of our personas have been identified too. Some designers complained our personas were lack of detailed information and they had to switch between personas and with the raw materials. How to create comprehensive personas with detailed information of users for ideation processes need to be considered in our further work.

\section{References}

1. Cooper, A.: The Inmates Are Running the Asylum, pp. 123-124. SAMS/Macmillan, Indianapolis, IA (1999)

2. Calde, S., Goodwin, K., Reimann, R.S.: Orcas: The First Integrated Information System for Long-term Healthcare Facility Management. In: Dykstra-Erickson, E. (ed.) Proceedings of Conference on Human Factors in Computing Systems, Minneapolis, Minnesota, USA, pp. 2-16. ACM Press, New York (2002)

3. Guojonsdottir, R., Lindquist, S.: Personas and Scenarios: Design Tool or A Communication Device. In: Proceedings of the 8th International Conference on the Design of Cooperative Systems, pp. 165-176 (2008)

4. Nielsen, L.: Engaging Personas and Narrative Scenarios. Vol. 17, PhD Series. Samfundslitteratur, Copenhagen, pp. 1-331 (2004)

5. Shah, J., Vargas-Hernandez, N.: Metrics for Measuring Ideation Effectiveness. Design Studies 24(2), 111-134 (2003)

6. Barki, H., Pinsonneault, A.: Small Group Brainstorming and Idea Quality: Is Electronic Brainstorming the Most Effective Approach? Small Group Research 32(2), 158-205 (2001) 